FINAL TECHNICAL REPORT FOR NASA GRANT NAG101084:

\author{
"WHIRL FLUTTER STABILITY OF TWO-BLADED \\ PROPROTOR/PYLON SYSTEMS IN HIGH SPEED FLIGHT"
}

AlFred E. GeSSOW Rotorcraft CENTER DEPARTMENT OF AEROSPACE ENGINEERING UNIVERSITY OF MARYLAND COLLEGE PARK, MD 20742 


\title{
WHIRL FLUTTER STABILITY OF TWO-BLADED PROPROTOR/PYLON SYSTEMS IN HIGH SPEED FLIGHT
}

\author{
Beerinder Singh * \\ Inderjit Chopra ${ }^{\dagger}$ \\ Alfred Gessow Rotorcraft Center \\ Department of Aerospace Engineering \\ University of Maryland at College Park, MD 20742
}

\begin{abstract}
The lack of polar symmetry in two-bladed rotors leads to equations of motion with periodic coefficients in axial flight, which is contrary to three or more bladed rotors that result in constant coefficient equations. With periodic coefficients, the analysis becomes involved, as a result very few studies have been directed towards the analysis of two-bladed rotors. In this paper, the aeroelastic stability of twobladed proprotor/pylon/wing combinations is examined in high speed axial flight. Several parametric studies are carried out to illustrate the special nature of two-bladed proprotors and to better understand the mechanism of whirl-flutter in such rotors. The wing beam bending mode for two-bladed rotors is found to be stable over the range of parameters examined, a behaviour very different from three-bladed rotors. Also, the wing torsion mode exhibits a new type of instability similar to a wing torsional divergence occuring at $1 / \mathrm{rev}$ frequency. This type of behaviour is not seen in three and more bladed rotors. The interaction between wing chordwise bending and torsion modes is found to be much greater in the case of two-bladed rotors and, over the range of parameters considered, these two modes govern the stability of the system.
\end{abstract}

\footnotetext{
- Graduate Research Assistant, Student Member AIAA

Alfred Gessow Professor and Director, Fellow AIAA Copyright (C)2002 The American Institute of Aeronautics and Astronautics Inc. All rights reserved
}

\section{Introduction}

Aeroelastic stability of a tiltrotor aircraft in forward flight has bern the subject of considerable research since the 1960's. In high speed axial flight, tiltrotors are subject to an instability known as proprotor whirl flutter. Due to high inflow through the rotor in this flight mode, large rotor forces dependent on the in-plane motion are generated. These forces are larger in tiltrotor aircraft than in conventional propeller driven aircraft due to large blade flap and lag motions in case of tiltrotors. With increasing airspeed, the rotor forces and the pylon/wing motion interact with each other to a point where the rotor forces become destabilizing and the rotor/pylon/wing system becomes unstable.

Most of the research on proprotor whirl flutter has focused on three or more blades. Several investigations of this phenomenon were conducted using pylon pivot models. Reed ${ }^{(1)}$ presented a review of these early efforts and highlighted some of the key issues associated with this instability. Johnson [2] developed a comprehensive mathematical model of a tiltrotor including a modal representation of the wing, and for high speed forward flight. Analytical results obtained with this analysis showed good correlation with full scale proprotor tests and the analysis was later extended to include elastic blades and helicopter and conversion modes of operation. Kvaternik and Kohn ${ }^{[3]}$ carried out 
an experimental parametric investigation of whirlflutter for a proprotor mounted on a rigid pylon with flexibility in pitch and yaw. Results of this study showed that proprotor whirl flutter can be predicted with linear stability analyses using two-dimensional, quasi-steady aerodynamics for the blade loading. Nixon ${ }^{[4,5]}$ conducted parametric studies for aeroelastic stability of a tiltrotor in forward flight using a finite element based comprehensive tiltrotor aeroelastic analysis. The effects of several design parameters such as rotor flap and lag frequencies, pitch-flap coupling, wing stiffness and sweep were examined to determine their effect on aeroelastic stability in the high speed axial flight mode. The rotor was assumed to be in a state of autorotation, i.e., rotor torque was not transferred to the wing and the wing vertical bending slope at the tip did not affect rotor inplane motion. Johnson ${ }^{[2]}$ showed that this assumption gives conservative estimates of whirl flutter stability. Lag frequency tuning was found to be a practical method for increasing flutter speed in axial flight and forward sweep of the wing was found to be destabilizing (Nixon ${ }^{[4,5]}$ ). The above models rely on experimental data to model the lag frequency variation with blade collective pitch and the distribution of blade flexibility inboard and outboard of the pitch bearing. To reduce the dependance on experimental data, Hathaway and Gandhi ${ }^{[6]}$ have recently made some refinements to the model which allow first principles based modeling of the lag frequency variation and blade flexibility distribution.

In recent years numerous approaches for the improvement of tiltrotor whirl mode boundaries for three-bladed tiltrotors have been investigated. Srinivas and Chopra ${ }^{[7]}$, Popelka ${ }^{[8]}$ et al., Corso[9] et al. and, Barkai and Rand ${ }^{[10]}$ investigated the use of aeroelastic tailoring of composite wing and rotor blades to enhance whirl mode stability of a tiltrotor in forward flight. Nixon ${ }^{[1]}$ et al. presented a review of the experimental and analytical efforts for stability augmentation of tiltrotors through aeroelastic tailoring of the wing and rotor blades. Srinivas ${ }^{[2]}$ et al. carried out studies on improving whirl mode stability of tiltrotors by using advanced geometry blades with tip sweep and tip anhedral/dihedral.

Acree $^{[13]}$ et al. investigated the effect of the chordwise positions of the rotor blade aerodynamic center and center of gravity on stability. Rearward offsets of the aerodynamic center with respect to the blade elastic axis and pitch axis were found to give large in- creases in the stability boundary, with the effect being most dominant for offsets at the outboard part of the blade. Acree ${ }^{[14]}$ also analyzed the effect of blade tip sweep on whirl mode stability of the V-22 tiltrotor with a tip mass extended on a boom forward of the leading edge to compensate for the aft shift of the tip center of gravity due to sweep.

All of the above studies were carried out for threebladed proprotors, although Johnson ${ }^{[2]}$ presented some results for a two-bladed propeller as well. Unlike a rotor with three or more blades, a two-bladed rotor lacks polar symmetry, which leads to proprotor equations of motion containing periodic coefficients. Thus, the stability analysis in the case of a two-bladed rotor is more involved and as a result, a lot less attention has been devoted to two-bladed configurations. In June 1998, whirl flutter was encountered during a flight test of the Pathfinder aircraft, fitted with several two-bladed propellers to be used on the Helios aircraft. These aircraft, built under the Environmental Research Aircraft and Sensor Technology (ERAST) program, have lightweight and highly fiexible wing structures and soft-mounted propellers. The simplified analysis for two-bladed rotors, when applied to the Pathfinder configuration with th: Helios propellers, was unable to predict the flutter encountered in 1998. Also, it is unclear whether design guidelines established for preventing whirl flutter instability for three or more blades can be applied to the two-bladed case also. The periodicity of the equations of motion for a two-bladed proprotor may introduce new phenomena that require careful examination and scrutiny. Hence a systematic study of whirl flutter of two-bladed proprotors is carried out to determine the effects of various design parameters on their stability.

\section{Analytical Model}

Figure 1(a) shows the coordinate systems used for the rotor and wing. The fixed frame rotor forces, which are transferred to the wing, are also shown. Figure 1(b) shows the geometry of the wing/pylon system. Flap and lag motions of the rotor blades are considered and, the blades are assumed to be rigid.

\subsection{Rotor and Hub Structural Model}

The equations of motion for a single rigid blade in the rotating frame with flap and lag degrees of freedom have been presented by Johnson ${ }^{[2]}$. These 


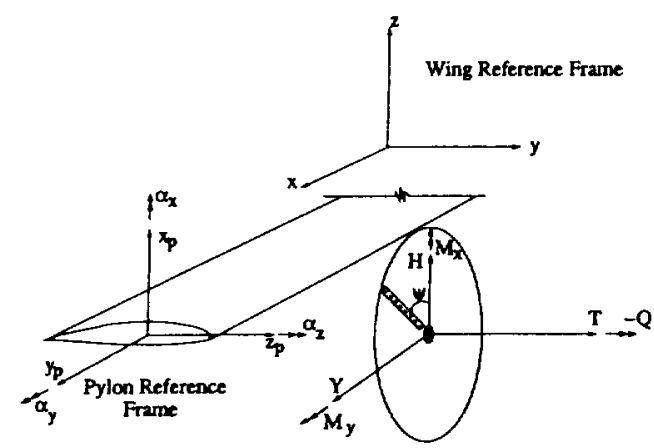

(a) Coordinate Systems for Rotor and Wing

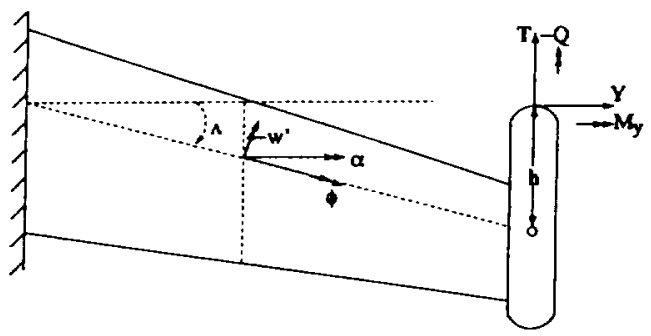

(b) Geometry of the Wing and Pylon

Figure 1: Coordinate Systems for Rotor/Wing and Wing Geometry

equations include the effects of pylon translation and rotation on the flap and lag motions,

$$
\begin{aligned}
& I_{\beta}^{*}\left(\stackrel{\beta}{\beta}^{(m)}+\nu_{\beta}^{2} \beta^{(m)}\right)+I_{\beta \alpha}^{*} l-\left(\stackrel{*}{\alpha}_{y}-2 \dot{\alpha}_{x}\right) \cos \psi_{m}+\left(\stackrel{*}{\alpha}_{x}\right. \\
& \left.\left.+2 \alpha_{y}^{*}\right) \sin \psi_{m}\right]+S_{\beta}^{* * *} z_{p}=\gamma \frac{M_{F}^{(m)}}{a c} \\
& I_{\zeta}^{*}\left(\stackrel{\zeta}{\zeta}^{(m)}+\nu_{\zeta}^{2} \zeta^{(m)}\right)+S_{\zeta}^{*}\left[\left(\stackrel{*}{x}_{p}+h \ddot{\alpha}_{y}\right) \sin \psi_{m}-\left({ }^{* *} y_{p}\right.\right. \\
& \left.\left.-h \alpha_{x}^{* *}\right) \cos \psi_{m}\right]-I_{\zeta \alpha}^{*}{ }^{* *}{ }_{z}=\gamma \frac{M_{L}^{(m)}}{a c}
\end{aligned}
$$

where $\beta^{(m)}, \zeta^{(m)}$ and $\psi_{m}$ are the flap motion, lag motion and the azimuthal position of the $m^{\text {th }}$ blade, $\nu_{\beta}$ and $\nu_{\zeta}$ are the non-dimensional flap and lag frequencies, respectively, and $\mathrm{h}$ is the distance between the pylon pivot point and the hub. $x_{p}, y_{p}$ and $z_{p}$ are pylon translational degrees of freedom, as shown in
Figure 1, while $\alpha_{x}, \alpha_{y}$ and $\alpha_{z}$ are pylon rotational degrees of freedom. $M_{F}^{(m)}$ and $M_{L}^{(m)}$ are respectively the aerodynamic flap and lag moments of the $m^{\text {th }}$ blade. The above equations can be written in matrix form as,

$$
\begin{aligned}
& \left.\left[I_{\beta}^{*}\right]\right]^{* *} \vec{\beta}_{R}+\left[K_{\beta}^{*}\right] \vec{\beta}_{R}+\left[I_{\beta P}^{*}\right]^{* *} \vec{p}+\left[C_{\beta P}^{*} \mid \vec{p}=\gamma \frac{\vec{M}_{F}}{a c}\right. \\
& {\left[I_{\zeta}^{*}\right] \vec{\zeta}_{R}^{* *}+\left[K_{\zeta}^{*}\right] \vec{\zeta}_{R}+\left[I_{\zeta P}^{*}\right]_{\vec{p}}^{* *}=\gamma \frac{\vec{M}_{L}}{a c}}
\end{aligned}
$$

where $\vec{\beta}_{R}$ and $\vec{\zeta}_{R}$ are vectors of the flap and lag motions in the rotating frame while $\vec{p}$ contains the pylon degrees of freedom. For $\mathrm{N}$ blades,

$$
\begin{gathered}
\ddot{\beta}_{R}=\left[\begin{array}{llll}
\beta^{(1)} & \beta^{(2)} & \ldots & \beta^{(N)}
\end{array}\right]^{T} \\
\ddot{\zeta_{R}}=\left[\begin{array}{lllll}
\zeta^{(1)} & \zeta^{(2)} & \ldots & \zeta^{(N)}
\end{array}\right]^{T} \\
\vec{p}==\left[\begin{array}{llllll}
x_{p} & y_{p} & z_{p} & \alpha_{x} & \alpha_{y} & \alpha_{x}
\end{array}\right]^{T}
\end{gathered}
$$

Other matrices in Equations 3 and 4 are defined in the Appendix. For coupling with the pylon/wing system, the above equations must be transformed to the fixed frame. Also, the pylon degrees of freedom must be replaced by the wing-tip degrees of freedom. This is accomplished using the following transformations,

$$
\vec{\beta}_{R}=\left[T_{R F} \mid \vec{\beta}_{F} \quad \vec{\zeta}_{R}=\left[T_{R F}\right] \vec{\zeta}_{F} \quad \vec{p}=\left[T_{\Lambda}\right] \vec{W}_{T i p}\right.
$$

where $\vec{\beta}_{F}$ and $\vec{\zeta}_{F}$ are the fixed frame rotor degrees of freedom, while $\vec{W}_{T_{\text {ip }}}$ is a vector containing the finite element degrees of freedom at the wing-tip. $\left[T_{R F}\right]$ is an $N \times N$ matrix relating the fixed frame rotor degrees of freedom to the rotating frame degrees of freedom, while $\left[T_{\Lambda}\right]$ is a transformation matrix that depends on the wing sweep $\Lambda$ and relates the pylon degrees of freedom to the wing-tip degrees of freedom. $\vec{\beta}_{F}, \vec{\zeta}_{F},\left[T_{R F}\right]$ and $\left[T_{\Lambda}\right]$ are as defined in the Appendix.

For a two-bladed rotor, the collective flap $\left(\beta_{0}\right)$, differential flap $\left(\beta_{1}\right)$, collective lag $\left(\zeta_{0}\right)$ and differential lag $\left(\zeta_{1}\right)$ motions comprise the fixed frame rotor degrees of freedom. In case of a three-bladed rotor, the fixed frame motions are collective flap $\left(\beta_{0}\right)$, cyclic flap $\left(\beta_{1 c}\right.$ and $\left.\beta_{1 s}\right)$, collective lag $\left(\zeta_{0}\right)$ and cyclic lag $\left(\zeta_{1 c}\right.$ and $\left.\zeta_{1 s}\right)$.

Note that, at this stage, the number of blades is assumed to be arbitrary so that the same analysis can be applie1 to both two and three-bladed rotors. 


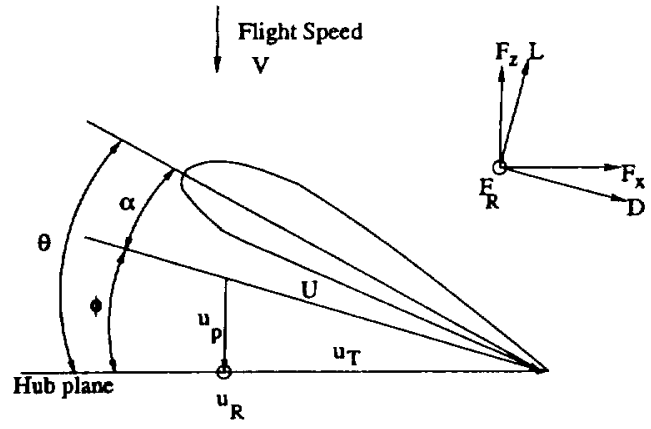

(a) Velocity and Force Components on a Typical Blade Section

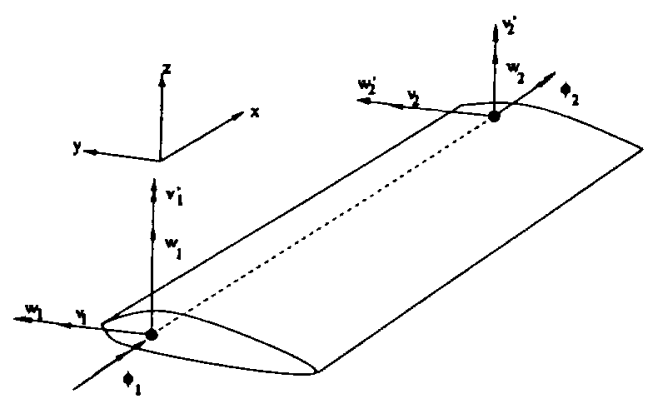

(b) Wing Element Degrees-of-Freedom

Figure 2: Blade Section Velocities and Wing Element

\subsection{Rotor Aerodynamics}

Figure 2(a) shows the velocity components at a blade section defined with respect to the hub plane. Aerodynamic forces are based on a linear strip theory and the rotor is assumed to be in a state of autorotation. The trim procedure is based on changing the collective pitch until the thrust becomes zero. Perturbations in the blade section velocities, for a forward flight speed $V$, can be written as,

$$
\begin{aligned}
& \delta u_{T}=r\left(\dot{\alpha}_{z}-\dot{\zeta}\right)+\left(-\dot{x}_{p}^{*} \sin \psi_{m}+\dot{y}_{p} \cos \psi_{m}-h\left(\alpha_{x}^{*}\right.\right. \\
&\left.\left.\cos \psi_{m}+\dot{\alpha}_{y} \sin \psi_{m}\right)+\frac{V}{\Omega R}\left(\alpha_{x} \cos \psi_{m}+\alpha_{y} \sin \psi_{m}\right)\right) \\
&=r \delta u_{T_{A}}+\delta u_{T_{B}}
\end{aligned}
$$

$$
\delta u_{p}=r\left(\dot{\beta}-\dot{*}_{y} \cos \psi_{m}+\dot{*}_{x} \sin \psi_{m}\right)+\dot{z}_{p}
$$

$$
\begin{aligned}
&=r \delta i \iota_{p_{B}}+\delta u_{p_{A}} \\
& \delta u_{R}=-i_{p}^{*} \sin \psi_{m}-\dot{x}_{p} \cos \psi_{m}+h\left(\alpha_{x}^{*} \sin \psi_{m}-*_{y}^{*}\right. \\
&\left.\cos \psi_{r}\right)-\frac{V}{\Omega R}\left(\alpha_{x} \sin \psi_{m}-\alpha_{y} \cos \psi_{m}\right)
\end{aligned}
$$

In Equations 5,6 and $7, \delta u_{T_{A}}, \delta u_{T_{B}}, \delta u_{p_{A}}, \delta u_{p_{B}}$ and $\delta u_{R}$ are independent of the radial position $r$ and hence they may be factored out of the integrands of the aerodynamic force integrals. The aerodynamic moments on a blade can then be written in terms of the aerodynamic coefficients $\left(M_{\mu}, M_{\zeta}\right.$ etc. $)$ and the perturbation velocities (see Johnson ${ }^{[2]}$ ). For example, the perturbation flap moment may be written as,

$$
\begin{aligned}
\delta \frac{M_{F}^{(m)}}{a c}= & M_{\mu} \delta u_{\tau_{B}}+M_{\zeta} \delta u_{T_{A}}+M_{\lambda} \delta u_{p_{A}}+M_{\dot{\beta}} \delta u_{p_{B}} \\
& +M_{\theta} \delta \theta
\end{aligned}
$$

where $\delta \theta$ is the perturbation in the collective angle. The perturtation velocities, $\delta u_{T_{A}}, \delta u_{p_{B}}$ etc., are functions of $\vec{\beta}_{R}, \vec{\zeta}_{R}, \vec{W}_{T i p}$ and $\psi_{m}$ for each blade, and thus the right hand side terms of Equations 3 and 4 can be expressed in terms of the system degrees of freedom and moved over to the left hand side of the equations.

\subsection{Wing Structural Model}

The wing structural model is based on a finite element formulation of an Euler-Bernoulli beam undergoing beamwise bending, chordwise bending and torsion, as shown in Figure 2(b). The axial degree of freedom is not considered since the wing is assumed to be rigid in axial direction.

\subsection{Wing Aerodynamics}

Wing aerodynamics is based on a quasi-steady approximation and the aerodynamic stiffness and damping matrices are determined and added to the corresponding structural matrices. Wing sweep (Figure 1(b)) is taken into consideration in formulating the wing aerodynamic matrices. 


\subsection{Coupling of Rotor/Wing System}

Coupling of the rotor/wing system essentially consists of two parts: (1) incorporating the rotor aerodynamic and inertial forces in the fixed frame into the wing model, and (2) incorporating the wing-tip degrees of freedom into the rotor equations of motion (see Sec. 2.1 and 2.2), this leads to a system of equations with periodic coefficients. In state space form, this system can be written as,

$$
\vec{y}=A(\psi) \vec{y}
$$

where ( ) denotes a derivative with respect to the azimuth, $A(\psi+2 \pi)=A(\psi)$ is a periodic matrix with period $2 \pi$ and $\vec{y}$ is a column vector of the $2 m$ system states.

\subsection{Stability Analysis}

The stability of the system defined by Equation 9 is examined using Floquet analysis. The Floquet transition matrix $\Phi(2 \pi)$, relates the states of the system at $\psi=0$ to the states at $\psi=2 \pi$,

$$
\vec{y}(2 \pi)=\Phi(2 \pi) \vec{y}(0)
$$

Each column of the Floquet transition matrix (FTM) is determined by integrating Equation 9 with a unit initial condition for one state and zero initial conditions for other states. Thus $2 m$ integrations need to be performed to obtain the FTM, where $2 m$ refers to the number of states. An effcient method, developed by Friedmann et.al. ${ }^{[15]}$, for computing the FTM in a single integration pass is used in the present study. Eigenvalues of the FTM are called Floquet multipliers and are denoted by $\theta_{i}(j=1,2 \ldots 2 m)$. The system is unstable if any of the Floquet multipliers have an absolute value larger than unity (see Johnson ${ }^{[16]}$, Nayfeh and Mook ${ }^{[17]}$ ). Floquet multipliers are related to the Floquet exponents $\left(\lambda_{i}\right)$ as,

$$
\lambda_{i}=\frac{1}{2 \pi} \ln \left(\theta_{i}\right)
$$

Real and imaginary parts of the Floquet exponents are related to the conventional damping $\left(\zeta_{i}\right)$ and frequency $\left(\omega_{p i}\right)$ respectively.

$$
\begin{aligned}
& \zeta_{i}=-\operatorname{Re}\left(\lambda_{i}\right)=-\frac{1}{2 \pi} \ln \left(\left|\theta_{i}\right|\right) \\
& \omega_{p i}=\operatorname{Im}\left(\lambda_{i}\right)=\frac{1}{2 \pi} \angle \theta_{i}
\end{aligned}
$$

\begin{tabular}{|r|c|}
\hline & Rotating Blade \\
& Freq. (per rev) \\
& In-vacuo \\
$\nu_{\beta}$ & 1.02 \\
$\nu_{\beta_{0}}$ & 1.85 \\
$\nu_{\zeta}$ & see Fig. 3 \\
$\nu_{\zeta_{0}}$ & 0 \\
\hline & Wing Freq. (per rev) \\
Beam & 0.42 \\
Chord & 0.70 \\
Torsion & 1.3 \\
\hline
\end{tabular}

Table 1: Frequencies of the Baseline System

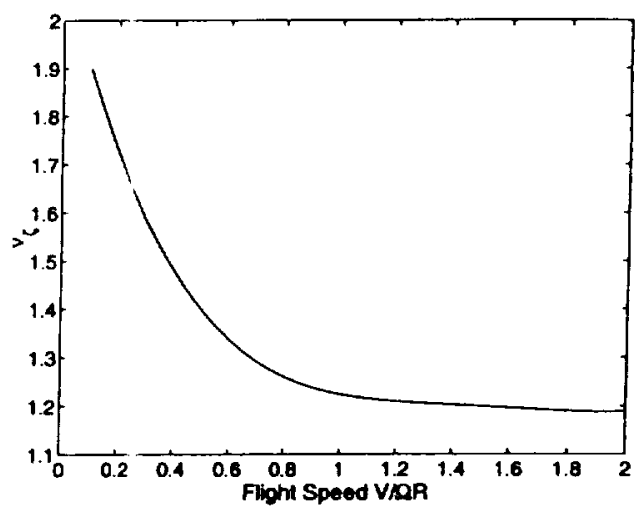

Figure 3: Blade Lag Frequency as a Function of Velocity

A subscript ' $\rho$ ' for the frequency indicates that only a principal value of the frequency can be determined due to the fact that the logarithm of a complex number has many branches, giving values for $\lambda_{i}$ that differ in frequency by integer multiples of the rotational speed $\Omega$. Compared to a constant coefficient system, the periodic system is also described by normal modes (i.e, eigenvectors) and the roots $\lambda_{i}$, but for a periodic: system the eigenvectors are periodic functions rather than constants (see Johnson ${ }^{[16]}$ ).

The analytical model was validated by comparing the frequency and damping variations with flight speed, with predicted results obtained by Nixon ${ }^{[4]}$ for a three bladed rotor. Also, the whirl flutter stability boundaries for a two-bladed propeller were compared with the results reported by Johnson ${ }^{[2]}$. In both cases, the present results matched with those of Nixon and Johnson. 


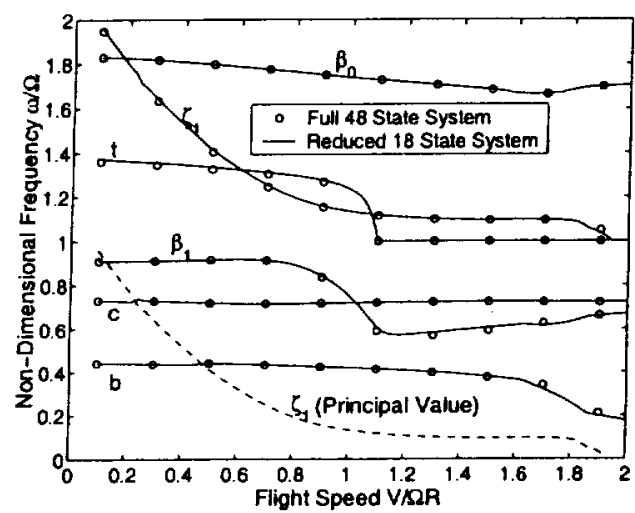

(a) Frequency

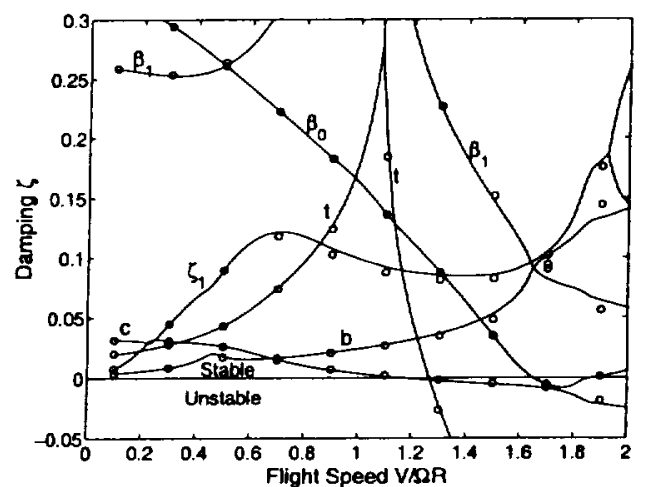

(b) Damping

Figure 4: Frequency and Damping as a Function of Velocity for Baseline System (Two-Bladed Rotor)

\section{Baseline System}

The present model is used to obtain the frequency and damping characteristics of two and three-bladed rotor/wing systems. The baseline rotor/wing system has the same properties as the baseline three-bladed rotor used by Nixon ${ }^{[4]}$. No wing sweep is considered in the present study. Table 1 shows the important in-vacuo frequencies of this system. The collective flap mode has a higher frequency because the blades act as cantilevers in this mode due to a gimballed hub. The in-vacuo lag frequency varies with collective pitch (and therefore flight speed) due to the distribution of lag flexibility inboard and outboard of

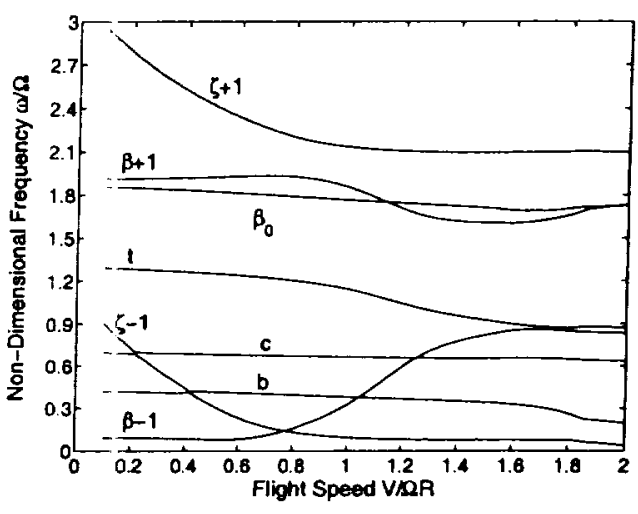

(a) Frequency

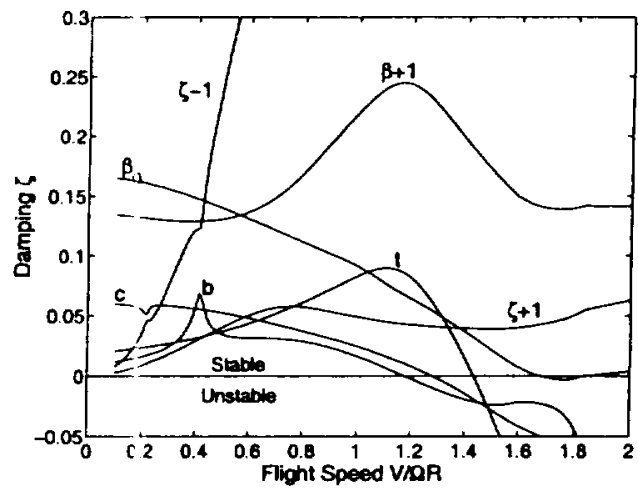

(b) Damping

Figure 5: Froquency and Damping as a Function of Velocity for Baseline System (Three-Bladed Rotor)

the pitch bearing (Figure 3 ). The rotor has a radius $\mathrm{R}=12.5 \mathrm{ft}$, Lock number $\gamma=3.83$, solidity $\sigma=0.089$, pitch-flap coupling $K_{p_{\beta}}=-0.268$ and rotational speed $\Omega=48 \mathrm{rad} / \mathrm{s}$. The wing $/$ pylon system has a span of $16.65 \mathrm{ft}$, a chord of $5.16 \mathrm{ft}$ and a pylon mast height of $4.28 \mathrm{ft}$. Three finite elements of length $4.55 \mathrm{ft}$ each are used to model the wing, with one pylon element of length $3.0 \mathrm{ft}$ at the tip. The wing has a beamwise stiffness $E I_{b}=3.13 \times 10^{7} \mathrm{lb}-\mathrm{ft}^{2}$, chordwise stiffness $E I_{c}=8.48 \times 10^{7} \mathrm{lb}-\mathrm{ft}^{2}$ and torsional stiffness $G J=1.62 \times 10^{7} \mathrm{lb}-\mathrm{ft}^{2}$.

Figure 4 shows the frequency and damping characteristics of th': baseline two-bladed rotor. It must be 
noted that the modes are highly coupled and hence their identification is based on the physical frequency and the behavior of periodic eigenvectors. In Figure 4, ' $b$ ', ' $c$ ' and ' $t$ ' refer to modes that are primarily wing beam, chord and torsion modes respectively, while ' $\beta_{0}$, ' $\beta_{1}$ ' and ' $\zeta_{1}$ ' are the rotor collective flap, differential flap and differential lag modes. The same nomenclature is used in all subsequent figures.

Shown in Figure 4 are the frequency and damping characteristics computed using two methods: (1) using a full 48 state system with 3 wing elements and 1 pylon element representing the wing, and (2) using an 18 state system obtained by applying static condensation to the wing equations and retaining only the five wing-tip degrees of freedom, prior to coupling with the rotor equations. The reduced system captures all the essential modes quite well and is computationally much faster than the full system. Further results in this paper are based on the reduced order system.

Figure 5 shows the frequency and damping characteristics of a three-bladed rotor having the same non-dimensional properties as the two-bladed rotor. As compared to the two-bladed rotor, two additional degrees of freedom are introduced in the three bladed rotor due to the flap and lag motions of the third blade. Thus for a three-bladed rotor, there are five important rotor modes $\left(\beta_{0}, \beta+1, \beta-1\right.$, $\zeta+1, \zeta-1)$ compared to three rotor $\operatorname{modes}\left(\beta_{0}, \beta_{1}\right.$ and $\zeta_{1}$ ) for the two-bladed case. In Figure $5, \beta-1$ and $\zeta-1$ refer to the low frequency flap and lag modes respectively, while $\beta+1$ and $\zeta+1$ are the high frequency flap and lag modes and $\beta_{0}$ is the collective flap mode. The collective lag mode $\zeta_{0}$ is not shown in Figures 4 and 5 since it has zero frequency due to the assumption that the rotor is in a state of autorotation.

A key difference between two and three-bladed rotor behaviour can be seen in the wing beam mode damping for the two cases. For the baseline threebladed rotor, the wing beam mode becomes unstable for $V / \Omega R>1.19$ as seen from the beam mode damping in Figure 5(b). However, it is evident from the beam mode damping for a two-bladed rotor (Figure 4(b)) that the wing beam mode remains stable for all flight speeds considered. An explanation of this behaviour is presented in Section 4.1.

For a three-bladed rotor, there is an energy transfer between the wing chord mode $c$, and the low frequency lag mode, $\zeta-1$, at the point where the $\zeta-1$ frequency crosses the chord mode frequency (Figure $5(\mathrm{~b}), V / \Omega R=0.2$ ). The chord mode damping reduces slightly at this point since the damping in the $\zeta-1$ mode is lower. A similar interaction is seen when the $\zeta-1$ mode frequency crosses the beam mode frequency at $V / \Omega R=0.4$ for the three-bladed rotor (Figure 5), where the beam mode damping shows a peak since, at this point, the damping in the $\zeta-1$ mode is higher than that in the beam mode. Figure 4 presents evidence of similar energy transfer between the differential lag mode $\zeta_{1}$ and the wing beam and chord modes for a two-bladed rotor. This occurs at the points where the principal value of the $\zeta_{1}$ mode frequency crosses the wing chord and beam mode frequencies at $V / \Omega R=0.22$ and 0.48 , respectively (Figure $4(\mathrm{a})$ ). As compared to a three-bladed rotor, the energy transfer between the rotor lag and wing modes is smaller in the case of a two-bladed rotor as indicated by the smaller peak in the beam mode damping for the two-bladed rotor (Figure 4(b)). Also, the valley in the chord mode damping at $V / \Omega R=0.22$ (Figure $4(\mathrm{~b})$ ) is very small for the two-bladed rotor. Unlike a three-bladed rotor the frequencies need not actually cross in case of a two-bladed rotor (i.e., only their principal values cross) since, for a two-bladed rotor, Floquet multipliers govern the interaction between the modes. For the two-bladed rotor the energy transfer occurs when both the real and imaginary parts of the Floquet multipliers of two modes approach each other.

In the case of a two-bladed rotor, for $V / \Omega R>1.1$, the Floquet multiplier for wing torsion becomes real, which causes a split in the damping curve for this mode, one part increasing and the other decreasing to become unstable at $V / \Omega R>1.25$ (Figure 4). This is a unique instability for a two-bladed rotor, similar to the $1 / \mathrm{rev}$ divergence of a two-bladed propeller (Johnson $^{(2)}$ ). The differential lag mode also shows similar behavior at a high flight speed (Figure 4, $V / \Omega R>1.9$ ). but remains stable.

\section{Parametric Studies}

Several parametric studies are carried out to determine the effect of key natural frequencies of the rotor and wing on the critical flight speed and to examine the interactions between various modes for a two-bladed rotor. The critical flight speed $V_{f}$ is the 


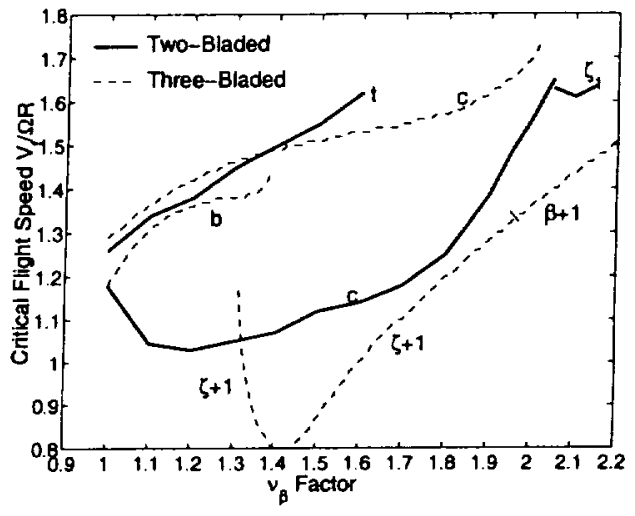

Figure 6: Effect of Flap Frequency $\left(\nu_{\beta}\right)$ on Critical Flight Speed

speed at which the damping for a particular mode becomes zero. For comparison, results of these parametric studies are plotted along with the results for a three bladed rotor having the same non-dimensional properties as the two-bladed rotor.

\subsection{Rotor Frequencies}

The rotor frequencies, $\nu_{\beta}$ and $\nu_{\zeta}$, are independently varied from their baseline values by factors varying between 1.0 and 2.2. It must be noted that extreme variations of in-vacuo flap and lag frequencies may not be practically possible, but they are of theoretical importance to establish the trends within the design range. Also, since the baseline lag frequency is a function of velocity as shown in Figure 3, the factor for $\nu_{\zeta}$ shifts the entire curve by a factor between 1.0 and 2.2. Since $\nu_{\beta}$ and $\nu_{\zeta}$ are uncoupled, in-vacuo flap and lag frequencies of the blades, an increase in these frequencies must primarily effect the $\beta_{1}$ or $\zeta_{1}$ frequencies which are the differential flap and lag frequencies of a coupled system with aerodynamics.

Figures 6 and 7 show the results of varying $\nu_{\beta}$ and $\nu_{\zeta}$ by the indicated factors from their baseline values. Dotted lines show the results for a three-bladed rotor having the same non-dimensional properties as the two-bladed rotor. Note that, in these figures, only the most critical modes for whirl stability are shown. Figures 8 and 9 , respectively show the frequency and damping characteristics for two and three-bladed rotors when $\nu_{\beta}$ is increased by a factor of 1.1 from its baseline value. Frequency and damping characteristics, with $\nu_{\beta}$ increased to 1.5 times its

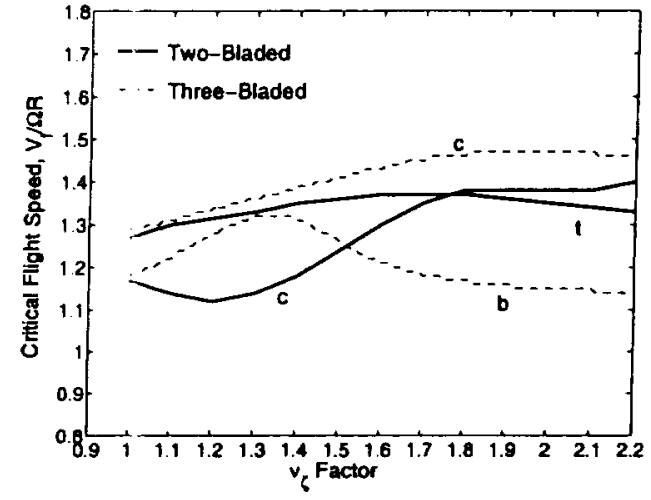

Figure 7: Effect of Lag Frequency $\left(\nu_{\zeta}\right)$ on Critical Flight Speed

baseline valıe, are shown in Figures 10 and 11 for two and thre-bladed rotors respectively. The principal observitions (italicized) are enumerated below along with their explanations (non-italicized).

1. For a two bladed rotor, the wing beam mode $b$ does not become unstable for the covered range of $\nu_{\beta}$ and $\nu_{\zeta}$. This is a key difference between two and threebladed rotors. It is evident from Figures 6 and 7 that the wing beam mode is quite critical for threebladed rotors, especially for low flap frequency, but it is stable for the two-bladed case.

For a three-bladed rotor, the wing beam mode becomes unstable due to its interaction with the $\beta-1$ and torsion modes (see Nixon ${ }^{[5]}$ ). As shown in Figures 5 and 9 , the $\zeta-1$ mode frequency acts as a barrier betwien the $\beta-1$ and beam mode frequencies at low flight speeds, but when the $\beta-1$ frequency crosses above the $\zeta-1$ frequency it drives the beam mode into an instability. As $\nu_{\beta}$ factor is increased bevond 1.4, the beam mode for a threebladed rotor does not become unstable as shown in Figure 6. Nxon ${ }^{[5]}$ showed that this is because of increased interaction between the rotor flap and lag modes and due to an increase in the wing torsion frequency which moves it further away from the wing beam mode frequency.

For a two-bladed rotor the differential flap $\left(\beta_{1}\right)$ frequency does not interact strongly with the wing beam mode frequency for the covered range of $\nu_{\beta}$ as shown in Figures 4,8 and 10 . Thus, the wing beam mode rimains stable for a two-bladed rotor.

2. As shown in Figure 6 the uing chord mode for a two-bladed rotor initially becomes less stable as $\nu_{\beta}$ 


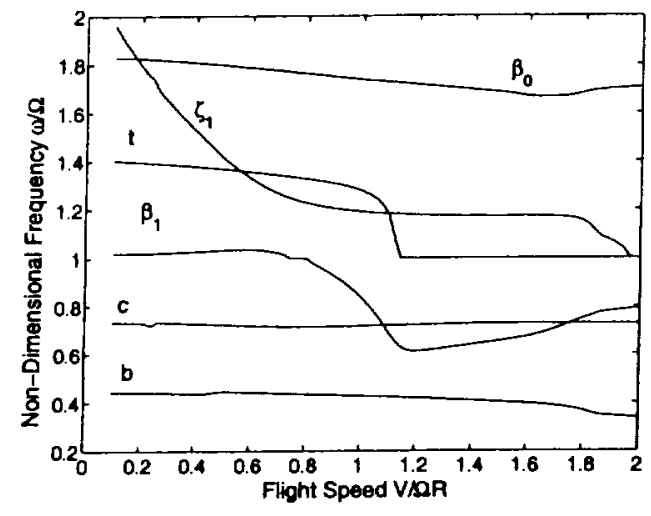

(a) Frequency

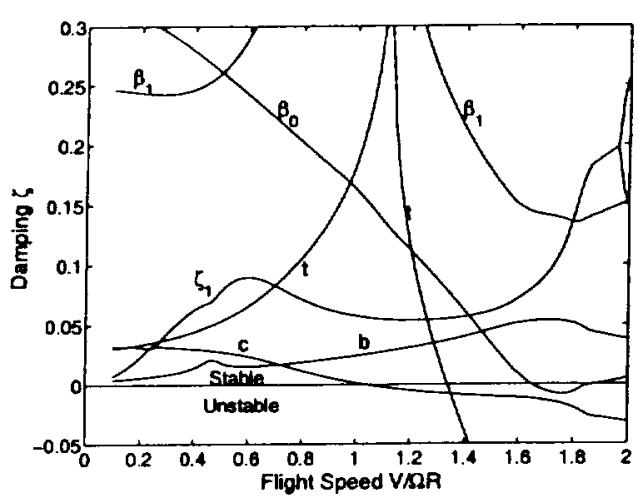

(b) Damping

Figure 8: Frequency and Damping Characteristics for $\nu_{\beta}$ Factor 1.1 (Two-Bladed Rotor)

is increased and then becomes more stable. Chord mode damping for a two-bladed rotor is affected by the placement of the $\beta_{1}, \zeta_{1}$ and wing torsion frequencies relative to the chord mode frequency. The $\beta_{1}$ frequency exerts a destabilizing influence on wing chord mode damping, whereas wing torsion frequency has a stabilizing influence on it. For low $\nu_{\beta}$ factors $\left(\nu_{\beta}<1.5\right)$, the $\zeta_{1}$ mode frequency falls below the torsion mode frequency for $V / \Omega R \sim 0.5-1.0$ (see Figures 4 and 8 ). In this range, the chord mode damping reduces faster and the overall effect is a slight reduction in critical flight speed for $\nu_{\beta}$ factors 1.1 and 1.2. For $\nu_{\beta}$ factors above 1.5, the $\zeta_{1}$

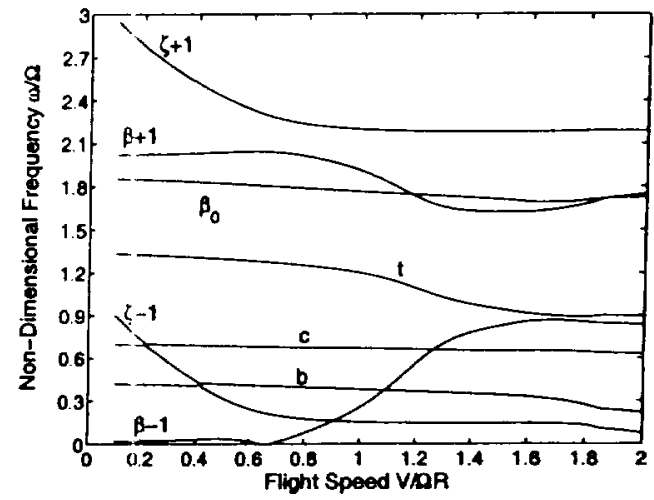

(a) Frequency

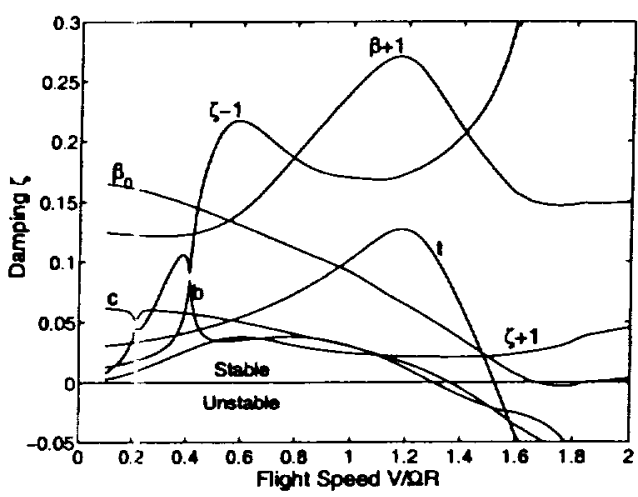

(b) Damping

Figure 9: Frequency and Damping Characteristics for $\nu_{\beta}$ Factor 1.1 (Three-Bladed Rotor)

mode frequency does not cross below the torsion mode frequency and also the $\beta_{1}$ mode frequency moves further away from the chord mode frequency for $V / \Omega R<1.1$ as shown in Figure 10. This stabilizes the wing chord mode at low flight speeds, but the increase in its critical flight speed is not significantly large since the $\beta_{1}$ mode frequency interacts very strongly with the chord mode at higher flight speeds (Figure 10(a)).

In case of a three-bladed rotor the wing chord mode gains greater stability as $\nu_{\beta}$ is increased, as shown in Figure 6 since, for a three-bladed rotor, the instability in the wing chord mode is caused by its 


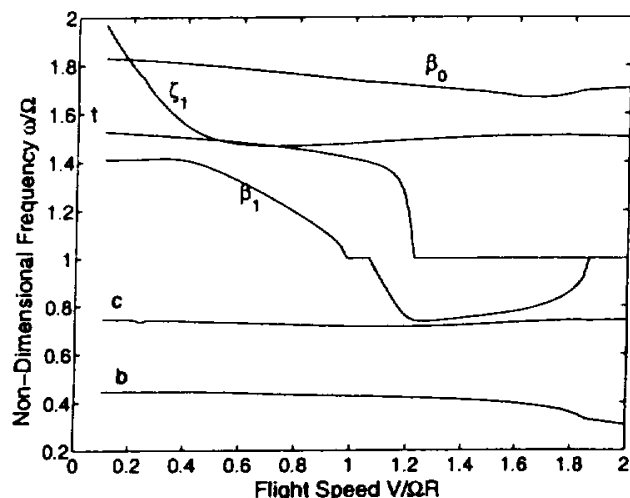

(a) Frequency

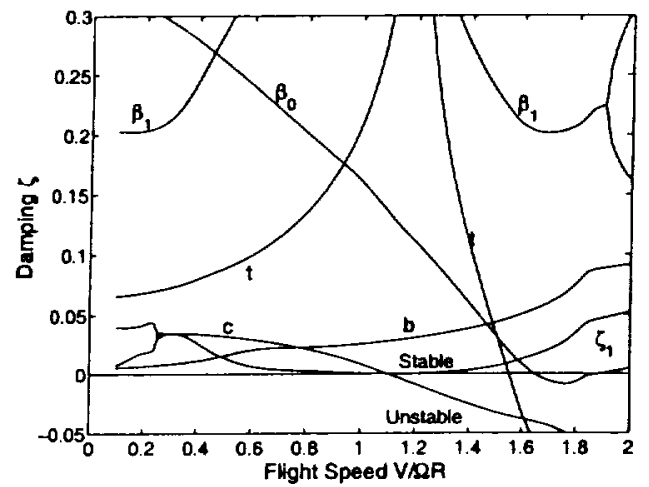

(b) Damping

Figure 10: Frequency and Damping Characteristics for $\nu_{\beta}$ Factor 1.5 (Two-Bladed Rotor)

interaction with the $\beta-1$ mode (Nixon ${ }^{[5]}$ ). As $\nu_{\beta}$ is increased, the $\beta-1$ mode frequency crosses above the $\zeta-1$ mode frequency at a higher flight speed (see Figures 5, 9 and 11). This leads to an increase in the critical flight speed for the wing chord mode in case of a three-bladed rotor.

From the above discussion it is clear that the wing chord mode is driven into an instability due to its interaction with the rotor flapping mode, for both two and three-bladed rotors and, in both cases, the placement of the rotor lag frequency is important. However, in case of a two-bladed rotor there is a significantly greater interaction between the wing chord

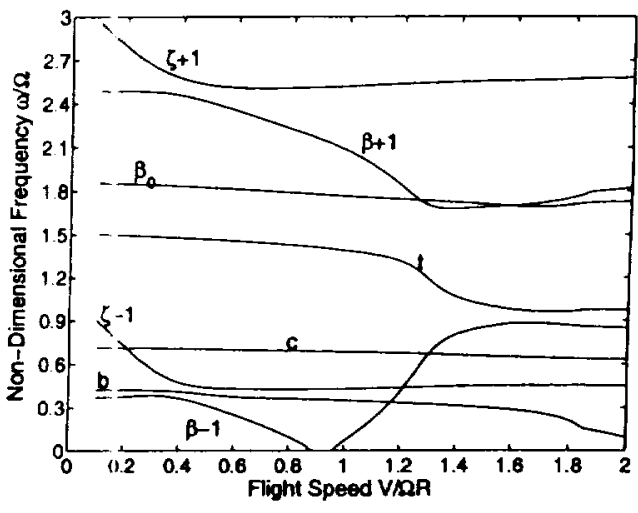

(a) Frequency

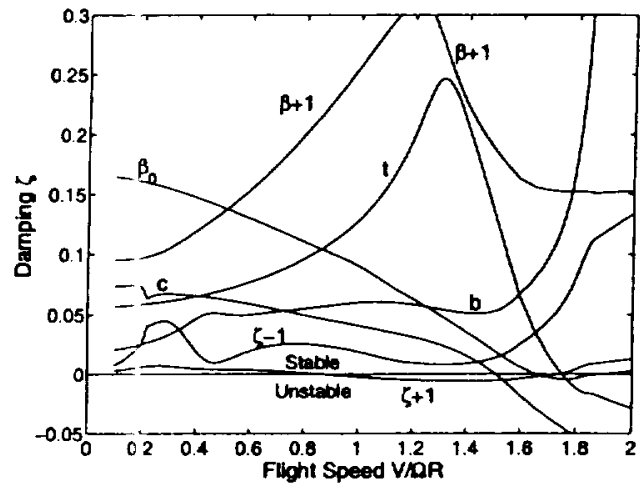

(b) Damping

Figure 11: Frequency and Damping Characteristics for $\nu_{\beta}$ Factor 1.5 (Three-Bladed Rotor)

and torsion modes. Further evidence of this interaction is preserited in Section 4.2.

3. As shown in Figure 6 , the wing chord mode for $a$ two-bladed rotor does not become unstable when $\nu_{\beta}$ factor is greater than 2.0, but an instability develops in the $\zeta_{1}$ mode at this point. For a two-bladed rotor, as the $\beta_{1}$ frequency increases, it has a lesser interaction with the chord mode and greater interaction with the $\zeta_{1}$ mode. Consequently, $\zeta_{1}$ mode damping reduces sharply as shown in Figures 4,8 and 10 .

For a three-bladed rotor also, there is a significant reduction in the damping for the $\zeta-1$ and $\zeta+1$ modes due to greater interactions with the rotor flap modes 


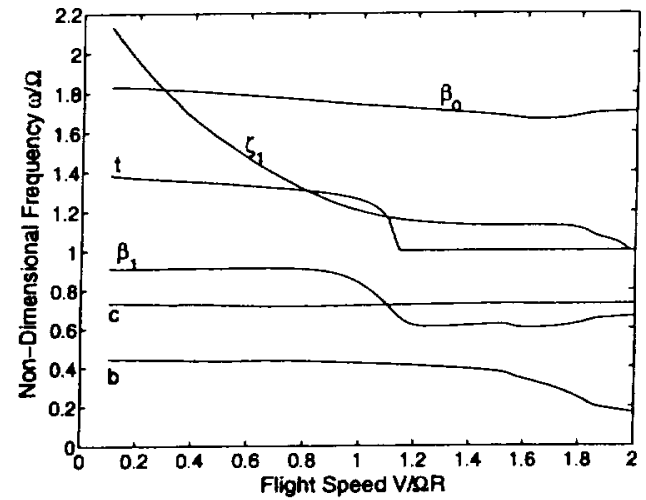

(a) Frequency

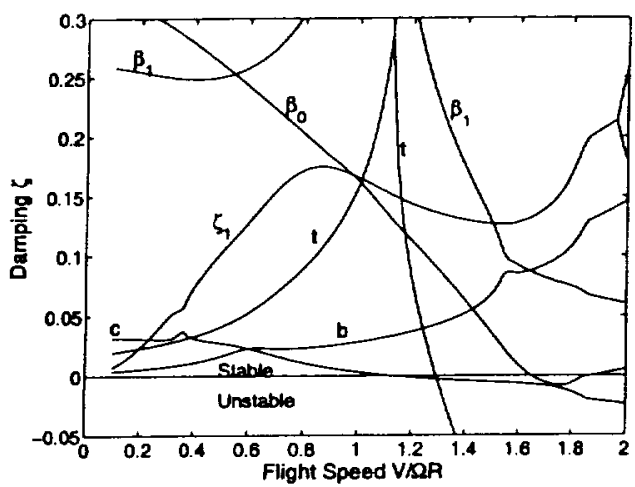

(b) Damping

Figure 12: Frequency and Damping Characteristics for $\nu_{\zeta}$ Factor 1.1 (Two-Bladed Rotor)

(see Figures 5, 9 and 11), as $\nu_{\beta}$ is increased. Such interaction leads to an instability in the highest frequency rotor mode (Nixon ${ }^{[5]}$ ). As shown in Figure 6 , for $\nu_{\beta}$ factors between 1.3 and 1.95 , the $\zeta+1$ mode is unstable in case of a three-bladed rotor. When $\nu_{\beta}$ factor increases above 1.95 , the $\beta+1$ mode becomes the rotor mode with the highest frequency and thus the instability shifts to the $\beta+1$ mode (Figure 6 ).

For a two-bladed rotor, the instability in the $\zeta_{1}$ mode occurs only when the $\nu_{\beta}$ factor is increased beyond 2.0 , in contrast to the three-bladed case where the $\zeta+1$ mode becomes unstable for $\nu_{\beta}$ factors greater

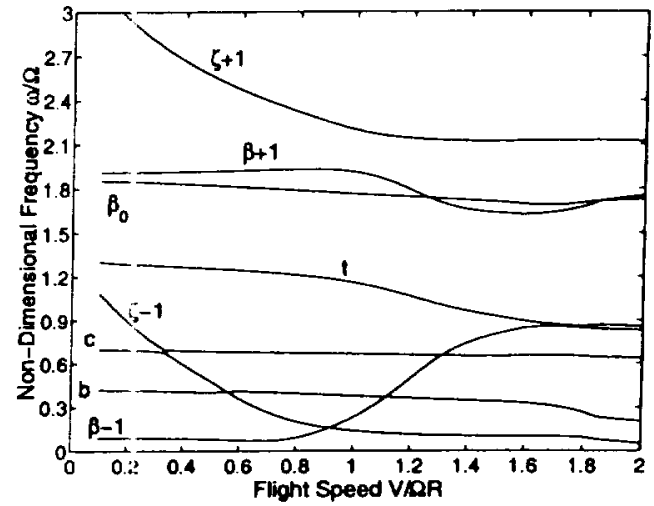

(a) Frequency

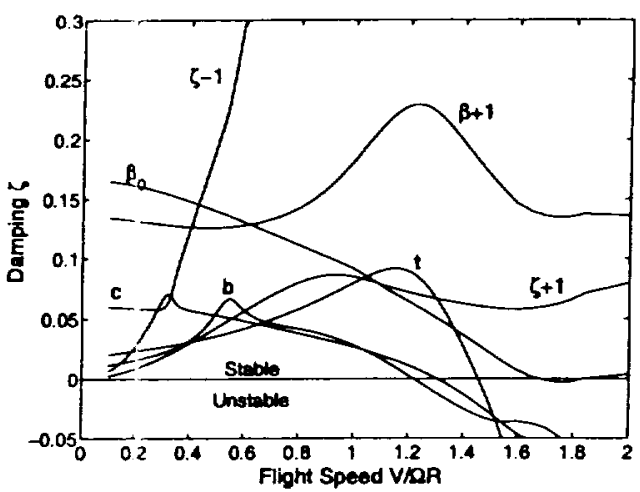

(b) Damping

Figure 13: Frequency and Damping Characteristics for $\nu_{\zeta}$ Factor 1.1 (Three-Bladed Rotor)

than 1.3 (Firgure 6). This is because for a twobladed rotor, at high values of $\nu_{\beta}$, the $\beta_{1}$ mode frequency has a close interaction with the $\zeta_{1}$ mode frequency only at low flight speeds (see Figure 10). At higher flight speeds, the $\beta_{1}$ mode frequency falls below $1 / \mathrm{rev}$ and interacts more with the wing chord mode. This stabilizes the $\zeta_{1}$ mode at higher speeds as shown in Figure 10(b). For a three-bladed rotor, the $\beta+1$ mode frequency is close to the $\zeta+1$ mode frequency even for the baseline case (Figure 5). Thus, only a small increase in $\nu_{\beta}$ is needed to increase the interaction between the $\zeta+1$ and $\beta+1$ modes and thus drive the $\zeta+1$ mode unstable in 


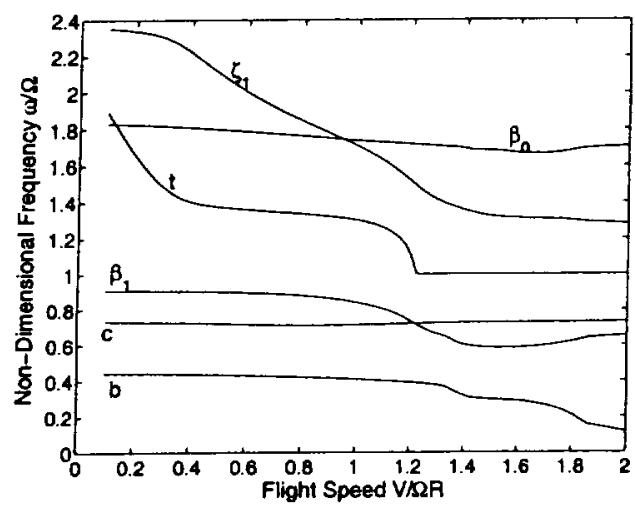

(a) Frequency

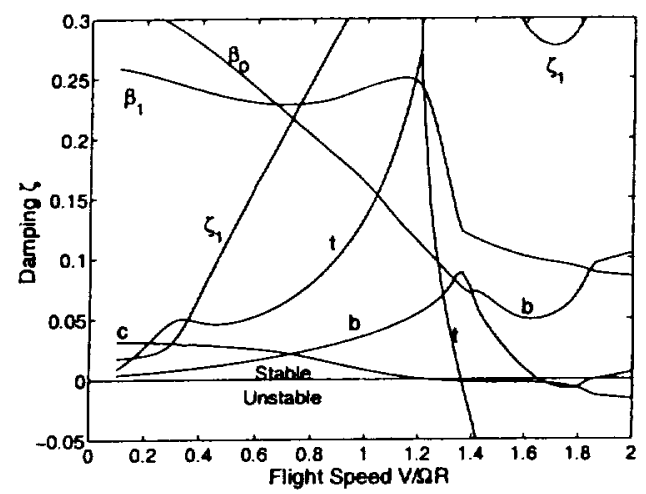

(b) Damping

Figure 14: Frequency and Damping Characteristics for $\nu_{\zeta}$ Factor 1.5 (Two-Bladed Rotor)

case of a three-bladed rotor, whereas a much larger increase in $\nu_{\beta}$ is required for the $\beta_{1}$ mode frequency of the two-bladed rotor to cause an instability in the $\zeta_{1}$ mode.

4. As shown in Figure 6 the wing torsion mode becomes more stable as $\nu_{\beta}$ factor increases and becomes completely stable for $\nu_{\beta}$ factors above 1.6. For a two-bladed rotor, as the $\beta_{1}$ frequency increases, it causes an increase in the wing torsion frequency as well (see Figures 4, 8, 10). This increases the flight speed at which the wing torsion mode diverges (i.e., speed at which the Floquet multiplier for torsion becomes real) and thus there is an increase in

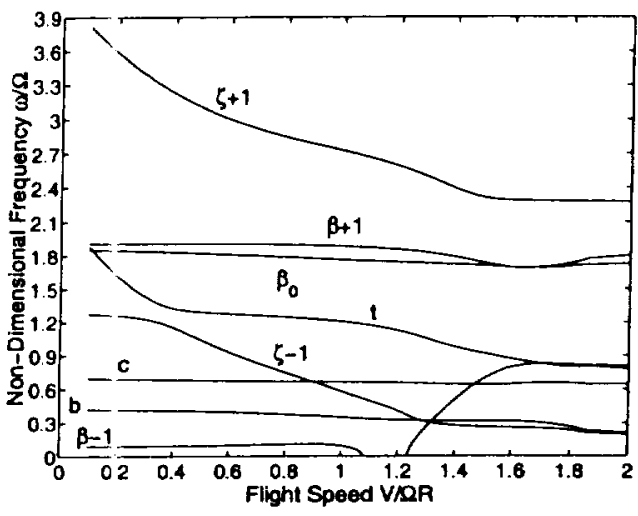

(a) Frequency

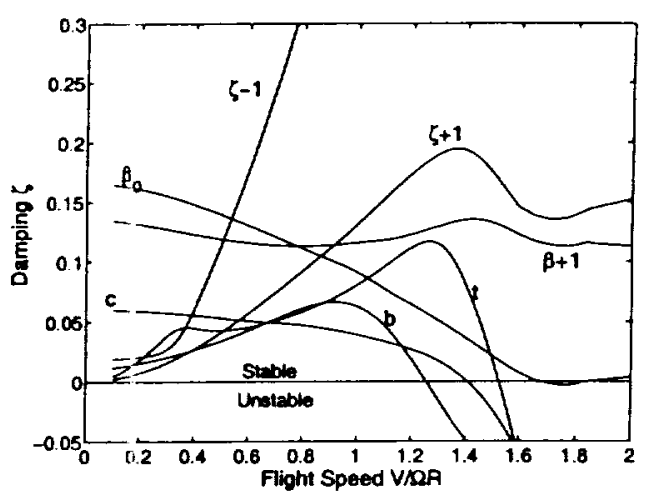

(b) Damping

Figure 15: Frequency and Damping Characteristics for $\nu_{\zeta}$ Factor 1.5 (Three-Bladed Rotor)

the critical Hight speed. Also, overall increase in the $\beta_{1}$ frequency causes it to rise above the chord mode frequency for $V / \Omega R>1.2$ (see Figure 10), and thus interact more with the torsion mode. This causes an increase in damping for the torsion mode at higher flight speeds, ultimately making this mode completely stable.

For a three-bladed rotor also, the wing torsion mode becomes more stable as $\nu_{\beta}$ is increased as seen from the torsion node damping in Figures 5, 9 and 11. This is because of an increase in torsion mode frequency which moves it further away from the $\beta-1$ mode frequency. 
5. For a two-bladed rotor, the wing chord mode initially becomes less stable as $\nu_{\zeta}$ factor is increased to 1.2 , then becomes more stable with $\nu_{\zeta}$ factor and $r e-$ mains relatively unaffected for $\nu_{\zeta}$ factors above 1.8 (Figure 7). There are three effects at work here. Firstly, an increase in the $\zeta_{1}$ frequency causes an increase in the flight speed where its principal value crosses the chord mode frequency. Thus the crossover now takes place at a point where the $\zeta_{1}$ mode damping is higher than the chord mode damping, resulting in a damping peak in the chord mode instead of a valley (Figure 12(b)). This peak shifts to higher flight speeds as $\nu_{\zeta}$ factor increases. Secondly, an increase in $\zeta_{1}$ frequency reduces the range of flight speeds over which it is below the torsion frequency (see Figures 4 and 12). This is a stabilizing effect for the chord mode as discussed before. Thirdly, the portion of the $\beta_{1}$ frequency below the chord frequency increases so that there is greater interaction between the $\beta_{1}$ and chord modes, which is destabilizing (Figures 12(a) and 14(a)). For $\nu_{\zeta}$ factors of 1.1 and 1.2 , the third effect is slightly greater than the first two leading to a slight decrease in the critical flight speed. For higher $\nu_{\zeta}$ factors, the first two factors cause an increase in the critical flight speed. For $\nu_{\zeta}$ factors above 1.8, the $\zeta_{1}$ frequency does not have a great effect on any other mode and thus the critical flight speed for the chord mode becomes relatively constant under the opposing influences of the $\beta_{1}$ and wing torsion modes.

For a three-bladed rotor, the wing chord mode becomes more stable as $\nu_{\zeta}$ is increased, until about $1.7 \nu_{\zeta}$, then remains relatively unaffected (Figure 7). For low values of $\nu_{\zeta}$, the damping in the wing chord mode for a three-bladed rotor is strongly influenced by the location of the $\zeta-1$ frequency (Nixon ${ }^{[5]}$ ). As $\nu_{\zeta}$ increases, there is an increase in the $\zeta-1$ fre quency as seen in Figure 15. This increases the flight speed at which the $\zeta-1$ mode frequency crosses the chord mode frequency, leading to an increase in the critical flight speed for the chord mode. Beyond a $\nu_{\zeta}$ factor of 1.7 , the $\zeta-1$ frequency moves above the wing torsion frequency and thus the flutter in the chord mode becomes dominated by the $\beta-1$ mode (Nixon ${ }^{[5]}$ ).

For both two and three-bladed rotors, at higher values of $\nu_{\zeta}$, the wing chord mode flutter becomes dominated by the flap mode. However, for low $\nu_{\zeta}$ the chord mode for a two-bladed rotor behaves differently due to the greater interaction between the wing torsion and chord modes for a two-bladed rotor.
6. As shoun in Figure 7, for a two-bladed rotor, the uing torsion mode stabilizes slightly as $\nu_{\zeta}$ factor increases to 1.7 and then destabilizes slightly for greater $\nu_{\zeta}$ factors. For both two and threebladed rotors, an increase in $\nu_{\zeta}$ causes an increase in the torsion mode frequency which is most pronounced at low flight speeds (Figures 14(a) and $15(\mathrm{a}), V / \Omega R<0.3)$. For a two-bladed rotor this leads to an increase in the flight speed at which the torsion mode diverges, i.e. the flight speed at which the torsion mode Floquet multiplier becomes real. This causes the split in the torsion mode damping to occur at a higher flight speed and thus there is an increase in the critical flight speed. As the $\nu_{\zeta}$ factor increases beyond 1.7, the effect of $\zeta_{1}$ frequency on the torsion frequency diminishes in the case of a two-bladed rotor. This causes a slight decrease in the flight speed at which the torsion mode diverges, leading to a slight decrease in the critical flight speed.

\subsection{Wing Frequencies}

Figures 16,17 and 18 show the variation of critical flight spted as the wing beam, chord and torsion stiffnesses (i.e. frequencies) are varied (independently) from their baseline values. Nixon ${ }^{15}$ showed that, for a three-bladed rotor, the stability of the wing beam mode depends on the proximity of the wing beam mode frequency to the wing torsion frequency. As shown in Figure 16, when the wing beam stiffness is reduced for a three-bladed rotor, the beam mode becomes more stable because the beam mode frequency moves away from the torsion frequency. Conversely, a reduction in the wing torsional stiffness for a three-bladed rotor brings the torsion mode frequency closer to the beam mode frequency, leading to a reduction in beam mode stability as shown in Figure 18. Nixon ${ }^{[5]}$ also showed that varying the wing frequencies causes a mild effect on wing chord mode stability for a three-bladed rotor because, in this case, the wing chord mode is not highly coupled to the other two wing modes (see Figures 16, 17 and 18).

For a two-bladed rotor, varying the wing beam frequency has a mild effect on the stability of the wing chord and torsion modes (Figure 16), since the beam mode does nit have much interaction with any of the other modes. When the frequency of the wing beam mode increases, it increases the flight speed at which the Floquet multiplier for the wing torsion 


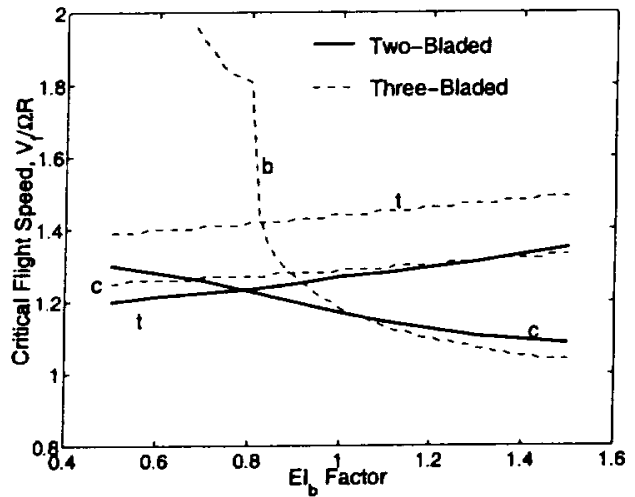

Figure 16: Effect of Wing Beam Stiffness $\left(E I_{b}\right)$ on Critical Flight Speed

mode becomes real. This leads to a small increase in the critical flight speed for the wing torsion mode of a two-bladed rotor. Also, greater interaction be tween the beam and torsion modes slightly reduces the stabilizing influence that the torsion mode exerts over the chord mode in the case of a two-bladed rotor. This leads to a slight decrease in the critical flight speed for the chord mode as wing beamwise stiffness $\left(E I_{b}\right)$ is increased.

The effects of wing chordwise stiffness $\left(E I_{c}\right)$ and wing torsional stiffness (GJ) on critical flight speed of a two-bladed system are more pronounced. Several observations regarding Figures 17 and 18 are enumerated below (italicized) along with their explanations (non-italicized).

1. As shown in Figure 17, the wing chord mode for a two-bladed rotor becomes more stable as its frequency is reduced. As discussed earlier, for a two-bladed rotor, wing chord mode damping depends on the placement of the $\beta_{1}, \zeta_{1}$ and wing torsion frequencies with respect to each other and also with respect to the wing chord mode frequency. As the chord mode frequency reduces, the $\beta_{1}$ and $\zeta_{1}$ frequencies move further away from the chord frequency for $V / \Omega R<1$. This increases the stability of the wing chord mode. 2. For a two-bladed rotor, increasing the wing chord mode frequency from its baseline value leads to a slight reduction in its critical fight speed initially (up to $E I_{c}$ factor 1.2), and then a slight increase takes place (Figure 17). As the wing chord mode frequency increases it comes closer to the torsion and $\beta_{1}$ frequencies for a two-bladed rotor. The net effect is a slight decrease in stability initially, due to

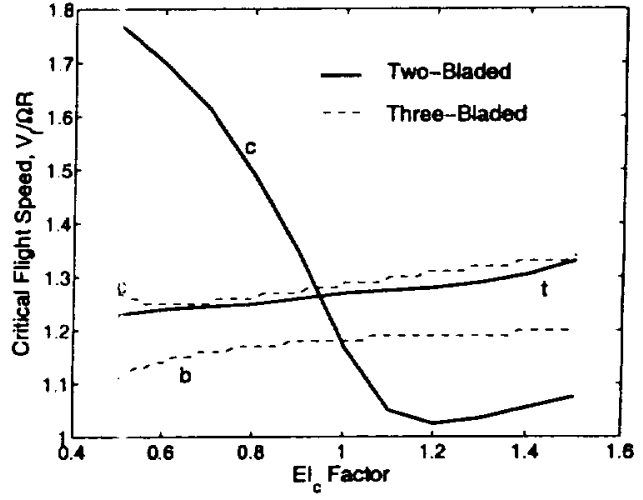

Figure 17: kffect of Wing Chordwise Stiffness $\left(E I_{c}\right)$ on Critical Flight Speed

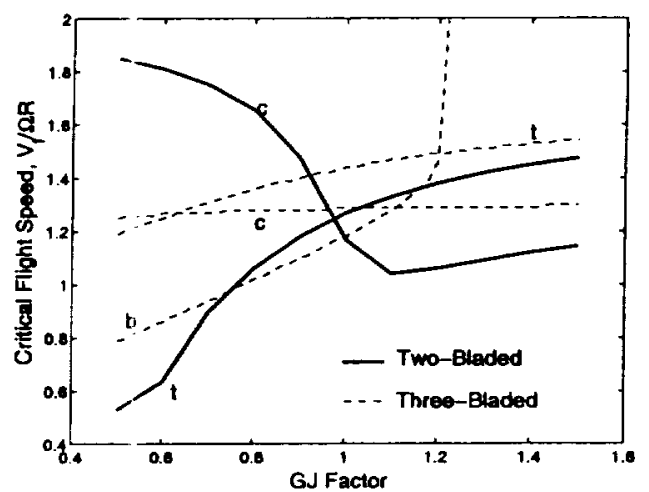

Figure 18: Effect of Wing Torsional Stiffness (GJ) on Critical Flight Speed

the influence of the $\beta_{1}$ mode, and then an increase due to the iuteraction between the wing chord and torsion modes.

3. Increasing the uning chord mode frequency has a small stabilizing influence on the uing torsion mode of a two-bladed system (Figure 10). In this case, as $E I_{c}$ increases, greater interaction between the wing chord and torsion modes causes a slight reduction in the rate at which the torsion mode damping reduces, beyond the point of divergence (i.e., the airspeed at which the Floquet multiplier for wing torsion becomes real). This causes a slight increase in the critical flight speed for the torsion mode.

4. As shown in Figure 18, a reduction in the wing torsion frequency from its baseline value causes a large increase in chord mode critical fight speed for 


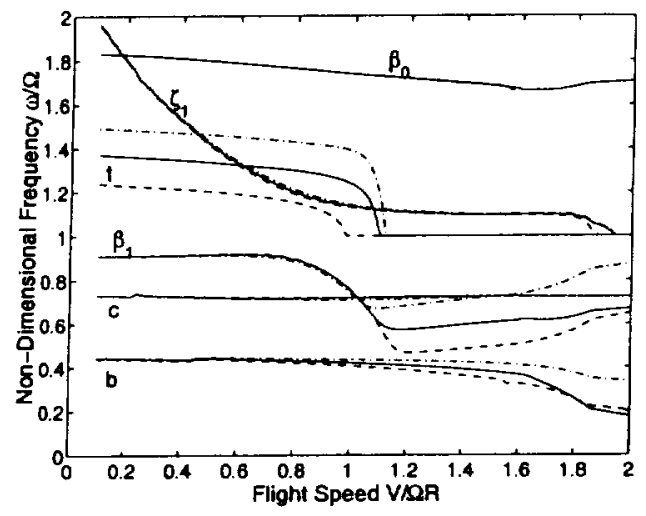

(a) Frequency

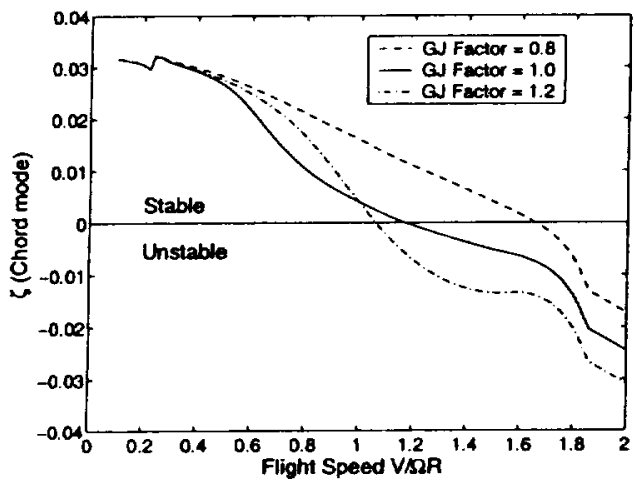

(b) Chord Mode Damping

Figure 19: Frequency and Chord Mode Damping Characteristics for Various GJ Factors (Two-Bladed Rotor)

a two-bladed system. There are two effects at work here. Firstly, as the torsion mode frequency reduces, the $\zeta_{1}$ frequency is unable to cross below it. This causes a significant reduction in the rate at which the chord mode damping reduces with flight speed, due to greater interaction between the torsion and chord modes (see Figure 19). Secondly, a reduction in torsion frequency reduces the portion of the $\beta_{1}$ frequency that lies below the chord frequency (see Figure $19, V / \Omega R>1$ ). This leads to reduced interaction between the $\beta_{1}$ and chord modes, leading to an increase in chord mode damping at higher flight

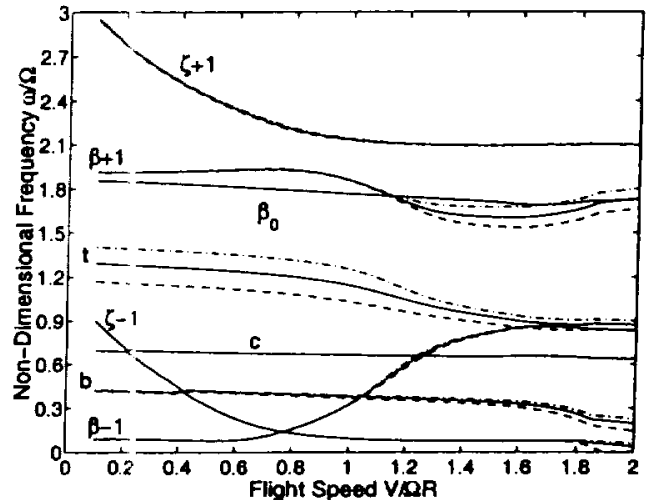

(a) Frequency

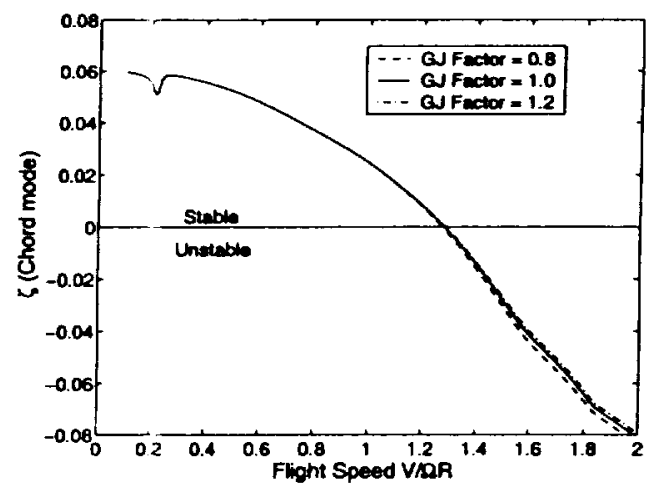

(b) Chord Mode Damping

Figure 20: Frequency and Chord Mode Damping Characteristics for Various GJ Factors (ThreeBladed Rotor)

speeds. The overall effect is a large increase in chord mode critical Alight speed for the two-bladed system, as $G J$ is reduced.

5. For a two-bladed rotor, a reduction in the wing torsion frequency significantly reduces the stability of the torsion mode (Figure 18). This is primarily a result of the loss in stiffness of the wing torsion mode, which reduces the airspeed at which the Floquet multiplier for wing torsion becomes real (i.e. the airspeed at which the wing torsion frequency reduces to $1 / \mathrm{rev}$ ). Also, as the torsion frequency reduces, the interaction between $\zeta_{1}$ and torsion modes 
reduces for low airspeeds $(V / \Omega R<0.5)$. This causes a reduction in torsion mode damping at low speeds. Consequently, when the torsion mode frequency becomes $1 / \mathrm{rev}$, it has a lower damping for low GJ factors. This causes a significant reduction in torsion mode stability.

Similar to a three-bladed system, the wing beam mode damping for a two-bladed rotor also reduces when $G J$ is reduced. This is because the wing beam mode is coupled to the wing torsion mode through the wing chordwise mass offset. However, for a twobladed rotor, the beam mode remains stable for most GJ factors, becoming unstable (at $V / \Omega R=1.24$ ) only when GJ is reduced by half (not shown in Figure 18).

The most important result of the wing frequency study is the greater coupling between the wing chord and torsion modes for a two-bladed rotor in contrast to the three-bladed case. Figures 16,17 and 18 show that wing chord and torsion modes are the governing modes for stability of a two-bladed proprotor. It is also evident that for a three-bladed rotor, changing the wing frequencies has little effect on chord mode stability. But for a two-bladed rotor, the effect of $E I_{c}$ factor and $G J$ factor on chord mode stability is quite pronounced. This indicates that there is greater interaction between chord and torsion modes for two-bladed rotors, as discussed in Section 4.1. A comparison of wing chord mode damping for two and three-bladed rotors (see Figures 19(b) and 20(b)), as affected by the wing torsional frequency, confirms the increased coupling of wing chord and torsion modes for two-bladed systems. In Figure 19(a), for low flight speeds $(V / \Omega R<1.0)$, only the wing torsion frequency is effected by the change in wing torsional stiffness. Thus, for the two-bladed system, the change in chord mode damping (Figure 19(b)) must be due to the interaction between the wing torsion and chord modes. In contrast, for the three-bladed system, the change in wing torsional frequency has only the slightest effect on chord mode damping (Figure 20), indicating a very weak coupling between wing chord and torsion modes.

\section{Conclusions}

The results of several parametric studies on the stability of two-bladed proprotors are presented. These studies illustrate the special nature of two-bladed rotors as opposed to three or multi-bladed rotors. Unlike a three-bladed rotor, the wing beam mode for a two-bladed rotor does not interact much with any of the other modes and thus remains stable. The wing torsion mode exhibits a unique instability similar to a wing torsi snal divergence at $1 / \mathrm{rev}$ frequency. Also, the interaction between the wing chord and torsion modes is much more pronounced in the case of twobladed rotors. It is shown that the stability of the wing chord mode is governed by a complex interaction between the rotor differential lap, differential lag and the wing torsion and chord modes. Also, the wing chord and torsion modes are the two modes that govern the stability of two-bladed proprotors over the range of parameters considered.

\section{Acknowledgements}

This research was sponsored by NASA Langley Research Center under Grant No. NAG 101084. Dr. A. Poto:zky was the technical monitor. The authors also thank Dr. R.G. Kvaternik (NASA Langley Research Center) for valuable technical discussions and Dr. Mark W. Nixon (Army/NASA Langley Research Center) for providing data and valuable suggestions.

\section{References}

[1] Reed, W. H., "Propeller-Rotor Whirl Flutter: A State of the Art Review," Journal of Sound and Vibration, Vol. 4, No. 3, 1966.

[2] Johnson, W., "Dynamics of Tilting Proprotor Aircraft in Cruise Flight," Tech. Rep. TN D7677, NASA, May 1974.

[3] Kvaternik, R. G. and Kohn, J. S., "An Experimental and Analytical Investigation of Proprotor Whirl Flutter," Tech. Paper TP 1047 , NASA, 1977.

[4] Nixon, M. W., Aeroelastic Response and Stability of Tiltrotors with Elastically Coupled Composite Rotor Blades, $\mathrm{Ph} . \mathrm{D}$. thesis, University of Marylani, College Park, 1993.

[5] Nixon, M. W., "Parametric Studies for Tiltrotor Aeronlastic Stability in High Speed Flight," Journal of the American Helicopter Society, Vol. 38, No. 4, October 1993.

[6] Hathaway, E. and Gandhi, F., "Modeling Refinements in Simple Tiltrotor Aeroelastic Stability Analyses," Presented at the 9th International Wirkshop on Aeroelasticity of Rotorcraft Systems, Ann Arbor, Michigan, October 2001. 
17] Srinivas, V. and Chopra, I., "Validation of a Comprehensive Aeroelastic Analysis for Tiltrotor Aircraft," Journal of the American Helicopter Society, Vol. 43, No. 4, October 1998.

[8] Popelka, D. A., Lindsay, D., Parham, T., Berry, V., and Baker, D. J., "Results of an Aeroelastic Tailoring Study for a Composite Tiltrotor Wing," Presented at the American Helicopter Society 51st Annual Forum, Fort Worth, Texas, May 1995.

[9] Corso, L. M., Popelka, D. A., and Nixon, M. W., "Design, Analysis and Test of a Composite Tailored Tiltrotor Wing," Presented at the American Helicopter Society 53rd Annual Forum, Virginia Beach, Virginia, April 1997.

[10] Barkai, S. M. and Rand, O., "The Influence of Composite Induced Couplings on Tiltrotor Whirl Flutter Stability," Journal of the American Helicopter Society, Vol. 43, No. 2, April 1998.

[11] Nixon, M., Piatak, D. J., Corso, L. M., and Popelka, D. A., "Aeroelastic Tailoring for Stability Augmentation and Performance Enhancements of Tiltrotor Aircraft," Journal of the American Helicopter Society, Vol. 45, No. 4, October 2000.

[12] Srinivas, V., Chopra, I., and Nixon, M. W., "Aeroelastic Analysis of Advanced Geometry Tiltrotor Aircraft," Journal of the American Helicopter Society, Vol. 43, No. 3, July 1998.

[13] Acree, C. W., Peyran, R. J., and Johnson, W., "Rotor Design for Whirl Flutter: An Examination of Options for Improving Tiltrotor Aeroelastic Stability Margins," Presented at the American Helicopter Society 55th Annual Forum, Montreal, Canada, May 1999.

[14] Acree, C. W, "Effects of Rotor Design Variations on Tiltrotor Whirl-Mode Stability," Presented at the Tiltrotor/Runway Independent Aircraft Technology and Applications Specialists' Meeting of the American Helicopter Society, Arlington, Texas, March 2001.

[15] Friedmann, P., Hammond, C. E., and Woo, T. H., "Efficient Numerical Treatment of $\mathrm{Pe}$ riodic Systems with Applications to Stability
Probleins," International Journal for Numerical Methods in Engineering, Vol. 11, 1977, pp. 1117-1136.

[16] Johnson, W., Helicopter Theory, Dover Publications. 1994.

(17) Nayfeh, A. H. and Mook, D. T., Nonlinear Oscillations, John Wiley, 1995.

\section{Appendix}

For an even number of blades,

$$
\begin{aligned}
& \vec{\beta}_{F}=\left[\begin{array}{lllllll}
\beta_{0} & \beta_{1 c} & \beta_{1 s} & \ldots & \beta_{M c} & \beta_{M s} & \beta_{N / 2}
\end{array}\right]^{T} \\
& \vec{\zeta}_{F}=\left[\begin{array}{lllllll}
\zeta_{0} & \zeta_{1 c} & \zeta_{1 s} & \ldots & \zeta_{M c} & \zeta_{M s} & \zeta_{N / 2}
\end{array}\right]^{T}
\end{aligned}
$$

For an odd number of blades,

$$
\begin{aligned}
& \vec{\beta}_{F}=\left[\begin{array}{llllll}
\beta_{0} & \beta_{1 c} & \beta_{1 s} & \ldots & \beta_{M c} & \beta_{M \cdot}
\end{array}\right]^{T} \\
& \vec{\zeta}_{F}=\left[\begin{array}{lllllll}
\zeta_{0} & \zeta_{1 c} & \zeta_{1 s} & \ldots & \zeta_{M c} & \zeta_{M s}
\end{array}\right]^{T}
\end{aligned}
$$

where,

$$
M= \begin{cases}\frac{N-2}{N^{2}}-1 & \text { if } N \text { is even } \\ \frac{N}{2} & \text { is odd }\end{cases}
$$

$$
\left[I_{\beta}^{*}\right]=\left[\begin{array}{cccc}
I_{\beta}^{*} & 0 & 0 & \ldots \\
0 & I_{\beta}^{*} & 0 & \ldots \\
0 & 0 & I_{\beta}^{*} & \ldots \\
\vdots & \vdots & \vdots & \ddots
\end{array}\right]_{N \times N}
$$

$$
\begin{aligned}
{\left[K_{\beta}^{*}\right] } & =\left[\begin{array}{cccc}
I_{\beta}^{*} \nu_{\beta}^{2} & 0 & 0 & \cdots \\
0 & I_{\beta}^{*} \nu_{\beta}^{2} & 0 & \cdots \\
0 & 0 & I_{\beta}^{*} \nu_{\beta}^{2} & \cdots \\
\vdots & \vdots & \vdots & \ddots
\end{array}\right]_{N \times N} \\
{\left[I_{\beta}^{*}\right] } & =\left[\begin{array}{ccccccc}
0 & 0 & S_{\beta}^{*} & I_{\beta \alpha}^{*} S_{1} & -I_{\beta \alpha}^{*} C_{1} & 0 \\
0 & 0 & S_{\beta}^{*} & I_{\beta \alpha}^{*} S_{2} & -I_{\beta \alpha}^{*} C_{2} & 0 \\
0 & 0 & S_{\beta}^{*} & I_{\beta \alpha}^{*} S_{3} & -I_{\beta \alpha}^{*} C_{3} & 0 \\
\vdots & \vdots & \vdots & \vdots & \vdots & \vdots \\
0 & 0 & S_{\beta}^{*} & I_{\beta \alpha}^{*} S_{N} & -I_{\beta \alpha}^{*} C_{N} & 0
\end{array}\right]_{N \times 6}
\end{aligned}
$$

where,

$$
S_{m}=\sin \psi_{m} \text { and } C_{m}=\cos \psi_{m}
$$




$$
\left[C_{\beta P}^{*}\right]=\left[\begin{array}{cccccc}
0 & 0 & 0 & 2 I_{\beta \alpha}^{*} C_{1} & 2 I_{\beta \alpha}^{*} S_{1} & 0 \\
0 & 0 & 0 & 2 I_{\beta \alpha}^{*} C_{2} & 2 I_{\beta \alpha}^{*} S_{2} & 0 \\
0 & 0 & 0 & 2 I_{\beta \alpha}^{*} C_{3} & 2 I_{\beta \alpha}^{*} S_{3} & 0 \\
\vdots & \vdots & \vdots & \vdots & \vdots & \vdots \\
0 & 0 & 0 & 2 I_{\beta \alpha}^{*} C_{N} & 2 I_{\beta \alpha}^{*} S_{N} & 0
\end{array}\right]_{N \times 6}
$$

(9)

$$
\left[T_{\Lambda}\right]=\left[\begin{array}{ccccc}
1 & 0 & 0 & 0 & 0 \\
0 & 0 & \sin \Lambda & 0 & 0 \\
0 & 0 & \cos \Lambda & 0 & 0 \\
0 & 0 & 0 & 1 & 0 \\
0 & -\sin \Lambda & 0 & 0 & \cos \Lambda \\
0 & -\cos \Lambda & 0 & 0 & -\sin \Lambda
\end{array}\right]
$$

$$
\begin{aligned}
{\left[I_{\zeta}^{*}\right] } & =\left[\begin{array}{cccc}
I_{\zeta}^{*} & 0 & 0 & \cdots \\
0 & I_{\zeta}^{*} & 0 & \cdots \\
0 & 0 & I_{\zeta}^{*} & \cdots \\
\vdots & \vdots & \vdots & \ddots
\end{array}\right]_{N \times N} \\
{\left[K_{\zeta}^{*}\right] } & =\left[\begin{array}{cccc}
I_{\zeta}^{*} \nu_{\zeta}^{2} & 0 & 0 & \ldots \\
0 & I_{\zeta}^{*} \nu_{\zeta}^{2} & 0 & \cdots \\
0 & 0 & I_{\zeta}^{*} \nu_{\zeta}^{2} & \cdots \\
\vdots & \vdots & \vdots & \ddots
\end{array}\right]_{N \times N} \\
{\left[I_{\zeta P}^{*}\right] } & =S_{\zeta}^{*}\left[\begin{array}{cccccc}
S_{1} & -C_{1} & 0 & h C_{1} & h S_{1} & \bar{r} \\
S_{2} & -C_{2} & 0 & h C_{2} & h S_{2} & \bar{r} \\
S_{3} & -C_{3} & 0 & h C_{3} & h S_{3} & \bar{r} \\
\vdots & \vdots & \vdots & \vdots & \vdots & \vdots \\
S_{N} & -C_{N} & 0 & h C_{N} & h S_{N} & \bar{r}
\end{array}\right]_{N \times 6}
\end{aligned}
$$

where, $\bar{r}=-\frac{I_{\delta a}^{*}}{S_{\dot{\zeta}}^{*}}$.

$$
\left[T_{R F}\right]=\left[\begin{array}{ccccc}
1 & \cos \psi_{1} & \sin \psi_{1} & \ldots & \sin M \psi_{1} \\
1 & \cos \psi_{2} & \sin \psi_{2} & \ldots & \sin M \psi_{2} \\
\vdots & \vdots & \vdots & \ddots & \vdots \\
1 & \cos \psi_{N} & \sin \psi_{N} & \ldots & \sin M \psi_{N}
\end{array}\right]
$$

The column $\left[\begin{array}{llll}-1 & 1 & \ldots & (-1)^{N}\end{array}\right]^{T}$ must be appended to $\left[T_{R F}\right]$ if $\mathrm{N}$ is even.

The wing-tip degrees of freedom are,

$$
\vec{W}_{T i p}=\left[\begin{array}{lllll}
w_{t} & w_{t}^{\prime} & v_{t} & v_{t}^{\prime} & \phi_{t}
\end{array}\right]^{T}
$$

Pylon degrees of freedom $(\vec{p})$ are given by,

$$
\vec{p}=\left[T_{\Lambda}\right] \vec{W}_{T i p}
$$

